\title{
Hot in the Tropics
}

The approach to clinical conundrums by an expert clinician is revealed through the presentation of an actual patient's case in an approach typical of a morning report. Similarly to patient care, sequential pieces of information are provided to the clinician, who is unfamiliar with the case. The focus is on the thought processes of both the clinical team caring for the patient and the discussant.

This icon represents the patient's case. Each paragraph that follows represents the discussant's thoughts

Arpana R. Vidyarthi, MD ${ }^{1,2 \star}$, Gurpreet Dhaliwal, MD,4, Bradley Monash, MD³, Koin Lon Shum, MD5, Joanne Lee, MBBS ${ }^{6}$, Aimee K. Zaas, MD, MHS ${ }^{7}$

${ }^{1}$ Duke-NUS Graduate Medical School, Singapore; '2Department of Medicine, National University Health System, Singapore; ${ }^{3}$ Department of Medicine, University of California, San Francisco, California; ${ }^{4}$ Medical Service, San Francisco VA Medical Center, San Francisco, California; ${ }^{5}$ Department of Internal Medicine, Singapore General Hospital, Singapore; ${ }^{6}$ Department of Haematology-Oncology, National University Cancer Institute, Singapore; ${ }^{7}$ Department of Medicine, Duke University School of Medicine, Durham, North Carolina.

A 42-year-old Malaysian construction worker with subjective fevers of 4 days' duration presented to an emergency department in Singapore. He reported nonproductive cough, chills without rigors, sore throat, and body aches. He denied sick contacts. Past medical history included chronic hepatitis $\mathrm{B}$ virus (HBV) infection. The patient was not taking any medications.

For this patient presenting acutely with subjective fevers, nonproductive cough, chills, aches, and lethargy, initial considerations include infection with a common virus (influenza virus, adenovirus, Epstein-Barr virus [EBV]), acute human immunodeficiency virus (HIV) infection, emerging infection (severe acute respiratory syndrome [SARS], Middle Eastern respiratory syndrome coronavirus [MERS-CoV] infection, avian influenza), and tropical infection (dengue, chikungunya). Also possible are bacterial infections (eg, with Salmonella typhi or Rickettsia or Mycoplasma species), parasitic infections (eg, malaria), and noninfectious illnesses (eg, autoimmune diseases, thyroiditis, acute leukemia, environmental exposures).

The patient's temperature was $38.5^{\circ} \mathrm{C}$; blood pressure, $133 / 73 \mathrm{~mm} \mathrm{Hg}$; heart rate, 95 beats per minute; respiratory rate, 18 breaths per minute; and oxygen saturation, $100 \%$ on ambient air. On physical examination, he appeared comfortable, and heart, lung, abdomen, skin, and extremities were normal. Laboratory test results included white blood cell (WBC) count, $4400 / \mu \mathrm{L}$ (with normal differential); hemoglobin, $16.1 \mathrm{~g} / \mathrm{dL}$; and platelet count,

\footnotetext{
*Address for correspondence and reprint requests: Arpana R. Vidyarthi, MD, Division of Advanced Internal Medicine, Department of Medicine, NUHS Tower Block, Level 10, National University Health System, 1E Kent Ridge Rd, Singapore 119228; Telephone: +65-9009-8011; Fax: +65-6872-4130; E-mail: arpana.vidyarthi@duke-nus.edu.sg

Received: May 8, 2016; Revised: October 13, 2016; Accepted: October 18, 2016
}

2017 Society of Hospital Medicine DOI 10.12788/jhm.2753
$207,000 / \mu \mathrm{L}$. Serum chemistries were normal. C-reactive protein (CRP) level was $44.6 \mathrm{mg} / \mathrm{L}$ (reference range, 0.2 $9.1 \mathrm{mg} / \mathrm{L}$ ), and procalcitonin level was $0.13 \mathrm{ng} / \mathrm{mL}$ (reference range, $<0.50 \mathrm{ng} / \mathrm{mL}$ ). Chest radiograph was normal. Dengue antibodies (immunoglobulin M, immunoglobulin G [IgG]) and dengue NS1 antigen were negative. The patient was discharged with a presumptive diagnosis of viral upper respiratory tract infection.

There is no left shift characteristic of bacterial infection or lymphopenia characteristic of rickettsial disease or acute HIV infection. The serologic testing and the patient's overall appearance make dengue unlikely. The low procalcitonin supports a nonbacterial cause of illness. CRP elevation may indicate an inflammatory process and is relatively nonspecific.

Myalgias, pharyngitis, and cough improved over several days, but fevers persisted, and a rash developed over the lower abdomen. The patient returned to the emergency department and was admitted. He denied weight loss and night sweats. He had multiple female sexual partners, including commercial sex workers, within the previous 6 months. Temperature was $38.5^{\circ} \mathrm{C}$. The posterior oropharynx was slightly erythematous. There was no lymphadenopathy. Firm, mildly erythematous macules were present on the anterior abdominal wall (Figure 1). The rest of the physical examination was normal.

Laboratory testing revealed WBC count, $5800 / \mu \mathrm{L}(75 \%$ neutrophils, $19 \%$ lymphocytes, $3 \%$ monocytes, $2 \%$ atypical mononuclear cells); hemoglobin, $16.3 \mathrm{~g} / \mathrm{dL}$; platelet count, $185,000 / \mu \mathrm{L}$; sodium, $131 \mathrm{mmol} / \mathrm{L}$; potassium, 3.4 $\mathrm{mmol} / \mathrm{L}$; creatinine, $0.9 \mathrm{mg} / \mathrm{dL}$; albumin, $3.2 \mathrm{~g} / \mathrm{dL}$; alanine aminotransferase (ALT), $99 \mathrm{U} / \mathrm{L}$; aspartate aminotransferase (AST), $137 \mathrm{U} / \mathrm{L}$; alkaline phosphatase (ALP), 63 $\mathrm{U} / \mathrm{L}$; and total bilirubin, $1.9 \mathrm{mg} / \mathrm{dL}$. Prothrombin time was 11.1 seconds; partial thromboplastin time, $36.1 \mathrm{sec}$ onds; erythrocyte sedimentation rate, $14 \mathrm{~mm} / \mathrm{h}$; and CRP, $62.2 \mathrm{mg} / \mathrm{L}$. 


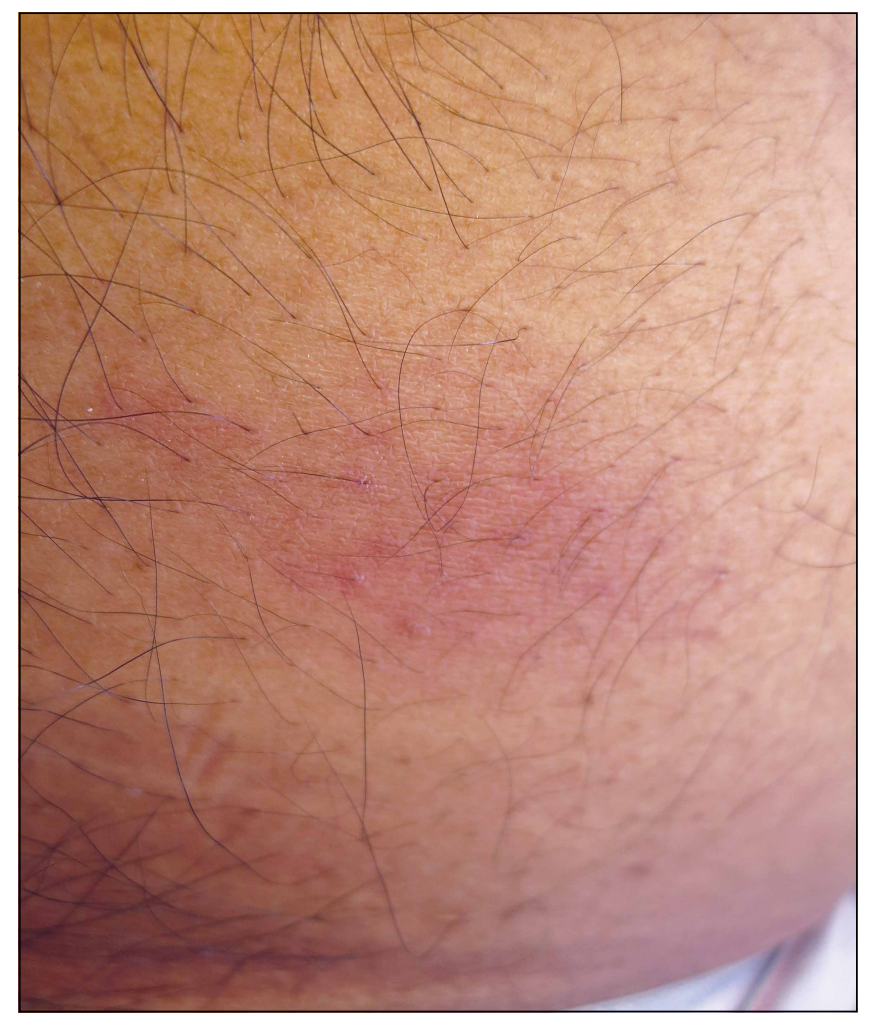

FIG. 1. Skin lesions on abdominal wall.

EBV, acute HIV, and cytomegalovirus infections often present with adenopathy, which is absent here. Disseminated gonococcal infection can manifest with fever, body aches, and rash, but his rash and the absence of penile discharge, migratory arthritis, and enthesitis are not characteristic. Mycoplasma infection can present with macules, urticaria, or erythema multiforme. Rickettsia illnesses typically cause vasculitis with progression to petechiae or purpura resulting from endothelial damage. Patients with secondary syphilis may have widespread macular lesions, and the accompanying syphilitic hepatitis often manifests with elevations in ALP instead of ALT and AST. The mild elevation in ALT and AST can occur with many systemic viral infections. Sweet syndrome may manifest with febrile illness and rash, but the acuity of this patient's illness and the rapid evolution favor infection.

The patient's fevers $\left(35^{\circ}-40^{\circ} \mathrm{C}\right)$ continued without pattern over the next 3 days. Blood and urine cultures were negative. Polymerase chain reaction (PCR) test of the nasal mucosa was negative for respiratory viruses. PCR blood tests for EBV, HIV-1, and cytomegalovirus were also negative. Antistreptolysin O (ASO) titer was 400 $\mathrm{IU} / \mathrm{mm}$ (reference range, $<200 \mathrm{IU} / \mathrm{mm}$ ). Antinuclear antibodies were negative, and rheumatoid factor was $12.4 \mathrm{U} / \mathrm{mL}$ (reference range, $<10.3 \mathrm{U} / \mathrm{mL}$ ). Computed tomography (CT) of the thorax, abdomen, and pelvis was normal. Results of a biopsy of an anterior abdominal wall skin lesion showed perivascular and periadnexal lymphocytic inflammation. Amoxicillin was started for the treatment of possible group A streptococcal infection.
PCR for HIV would be positive at a high level in acute HIV. The skin biopsy is not characteristic of Sweet syndrome, which typically shows neutrophilic infiltrate without leukocytoclastic vasculitis, or of syphilis, which typically shows a plasma cell infiltrate.

The patient's erythematous oropharynx may indicate recent streptococcal pharyngitis. The fevers, elevated ASO titer, and CRP level are consistent with acute rheumatic fever, but arthritis, carditis, and neurologic manifestations are lacking. Erythema marginatum manifests on the trunk and limbs as macules or papules with central clearing as the lesions spread outward - and differs from the patient's rash, which is firm and restricted to the abdominal wall.

Fevers persisted through hospital day 7. The WBC count was $1100 / \mu \mathrm{L}$ ( $75.7 \%$ neutrophils, $22.5 \%$ lymphocytes), hemoglobin was $10.3 \mathrm{~g} / \mathrm{dL}$, and platelet count was $52,000 / \mu \mathrm{L}$. Additional laboratory test results included ALP, 234 U/L; ALT, 250 U/L; AST, 459 U/L; lactate dehydrogenase, $2303 \mathrm{U} / \mathrm{L}$ (reference range, 222-454 $\mathrm{U} / \mathrm{L}$ ); and ferritin, $14,964 \mathrm{ng} / \mathrm{mL}$ (reference range, 47 $452 \mathrm{ng} / \mathrm{mL}$ ).

The duration of illness and negative diagnostic tests for infections increases suspicion for a noninfectious illness. Conditions commonly associated with marked hyperferritinemia include adult-onset Still disease (AOSD) and hemophagocytic lymphohistiocytosis (HLH). Of the 9 AOSD diagnostic (Yamaguchi) criteria, 5 are met in this case: fever, rash, sore throat, abnormal liver function tests, and negative rheumatologic tests. However, the patient lacks arthritis, leukocytosis, lymphadenopathy, and hepatosplenomegaly. Except for the elevated ferritin, the AOSD criteria overlap substantially with the criteria for acute rheumatic fever, and still require that infections be adequately excluded. HLH, a state of abnormal immune activation with resultant organ dysfunction, can be a primary disorder, but in adults more often is secondary to underlying infectious, autoimmune, or malignant (often lymphoma) conditions. Elevated ferritin, cytopenias, elevated ALT and AST, elevated CRP and erythrocyte sedimentation rate, and elevated lactate dehydrogenase are consistent with HLH. The HLH diagnosis can be more firmly established with the more specific findings of hypertriglyceridemia, hypofibrinogenemia, and elevated soluble CD25 level. The histopathologic finding of hemophagocytosis in the bone marrow, lymph nodes, or liver may further support the diagnosis of HLH.

Rash and fevers persisted. Hepatitis A, hepatitis C, Rickettsia IgG, Burkholderia pseudomallei (the causative organism of melioidosis), and Leptospira serologies, as well as PCR for herpes simplex virus and parvovirus, were all negative. Hepatitis B viral load was $962 \mathrm{IU} / \mathrm{mL}(2.98$ $\log )$, hepatitis $B$ envelope antigen was negative, and hepatitis B envelope antibody was positive. Orientia tsutsugamushi (organism responsible for scrub typhus) IgG titer was elevated at 1:128. Antiliver kidney microsomal antibodies and 


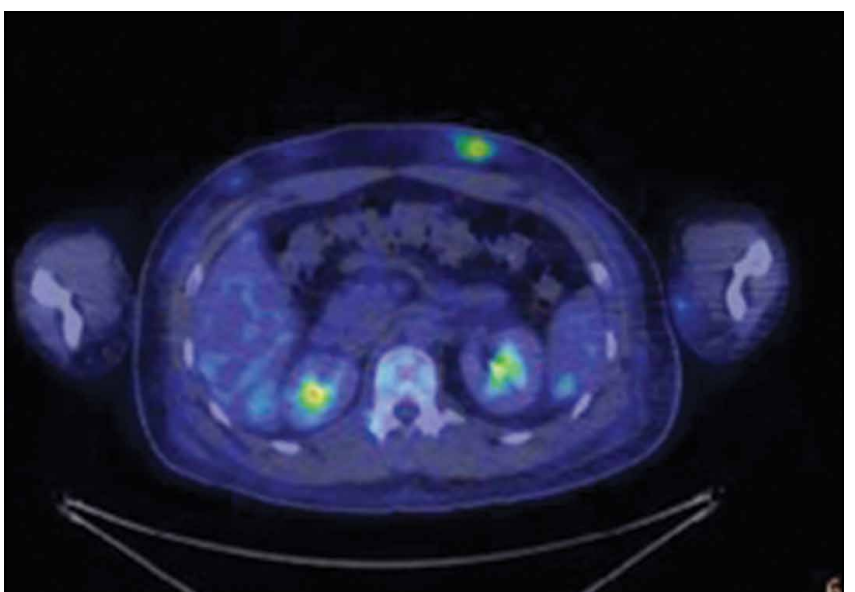

FIG. 2. Positron emission tomography computed tomography shows multiple fluorodeoxyglucose-avid cutaneous lesions (green) with surrounding patchy foci of subcutaneous fat stranding (blue-grey) in anterior abdominal wall and upper left arm, compatible with areas of lymphomatous infiltrates.

antineutrophil cytoplasmic antibodies were negative. Fibrinogen level was $0.69 \mathrm{~g} / \mathrm{L}$ (reference range, 1.8-4.8 $\mathrm{g} / \mathrm{L}$ ), and beta-2 microglobulin level was $5078 \mathrm{ng} / \mathrm{mL}$ (reference range, 878-2000 $\mathrm{ng} / \mathrm{mL}$ ). Bone marrow biopsy results showed hypocellular marrow with suppressed myelopoiesis, few atypical lymphoid cells, and few hemophagocytes. Flow cytometry was negative for clonal B lymphocytes and aberrant expression of $\mathrm{T}$ lymphocytes. Bone marrow myobacterial PCR and fungal cultures were negative.

The patient's chronic HBV infection is unlikely to be related to his presentation given his low viral load and absence of signs of hepatic dysfunction. Excluding rickettsial disease requires paired acute and convalescent serologies. $\mathrm{O}$ tsutsugamushi, the causative agent of the rickettsial disease scrub typhus, is endemic in Malaysia; thus, his positive $\mathrm{O}$ tsutsugamushi IgG may indicate past exposure. His fevers, myalgias, truncal rash, and hepatitis are consistent with scrub typhus, but he lacks the characteristic severe headache and generalized lymphadenopathy. Although eschar formation with evolution of a papular rash is common in scrub typhus, it is often absent in the variant found in Southeast Asia. Although elevated $\beta_{2}$ microglobulin level is used as a prognostic marker in multiple myeloma and Waldenström macroglobulinemia, it can be elevated in many immune-active states. The patient likely has HLH, which is supported by the hemophagocytosis seen on bone marrow biopsy, and the hypofibrinogenemia. Potential HLH triggers include $\mathrm{O}$ tsutsugamushi infection or recent streptococcal pharyngitis.

A deep-punch skin biopsy of the anterior abdominal wall skin lesion was performed because of the absence of subcutaneous fat in the first biopsy specimen. The latest biopsy results showed irregular interstitial expansion of medium-size lymphocytes in a lobular panniculated pattern. The lymphocytes contained enlarged, irregularly contoured nucleoli and were positive for T-cell markers CD2 and CD3 with reduction in CD5 expression. The lymphomatous cells were of CD8+ with uniform expression of activated cytotoxic granule protein granzyme $B$ and were positive for T-cell hemireceptor $\beta$.

Positron emission tomography (PET) CT, obtained for staging purposes, showed multiple hypermetabolic subcutaneous and cutaneous lesions over the torso and upper and lower limbs-compatible with lymphomatous infiltrates (Figure 2). Examination, pathology, and imaging findings suggested a rare neoplasm: subcutaneous panniculitis-like T-cell lymphoma (SPTCL). SPTCL was confirmed by $\mathrm{T}$-cell receptor gene rearrangements studies.

HLH was diagnosed on the basis of the fevers, cytopenias, hypofibrinogenemia, elevated ferritin level, and evidence of hemophagocytosis. SPTCL was suspected as the HLH trigger.

The patient was treated with cyclophosphamide, hydroxydoxorubicin, vincristine, and prednisone. While on this regimen, he developed new skin lesions, and his ferritin level was persistently elevated. He was switched to romidepsin, a histone deacetylase inhibitor that specifically targets cutaneous T-cell lymphoma, but the lesions continued to progress. The patient then was treated with gemcitabine, dexamethasone, and cisplatin, and the rashes resolved. The most recent PET-CT showed nearly complete resolution of the subcutaneous lesions.

\section{DISCUSSION}

When residents or visitors to tropical or sub-tropical regions, those located near or between the Tropics of Cancer and Capricorn, present with fever, physicians usually first think of infectious diseases. This patient's case is a reminder that these important first considerations should not be the last.

Generating a differential diagnosis for tropical illnesses begins with the patient's history. Factors to be considered include location (regional disease prevalence), exposures (food/ water ingestion, outdoor work/recreation, sexual contact, animal contact), and timing (temporal relationship of symptom development to possible exposure). Common tropical infections are malaria, dengue, typhoid, and emerging infections such as chikungunya, avian influenza, and Zika virus infection. ${ }^{1}$

This case underscores the need to analyze diagnostic tests critically. Interpreting tests as simply positive or negative, irrespective of disease features, epidemiology, and test characteristics, can contribute to diagnostic error. For example, the patient's positive ASO titer requires an understanding of disease features and a nuanced interpretation based on the clinical presentation. The erythematous posterior oropharynx prompted concern for postinfectious sequelae of streptococcal pharyngitis, but his illness was more severe and more prolonged than is typical of that condition. The isolated elevated O tsutsugamushi IgG titer provides an example of the role of epidemiology in test interpretation. Although a single positive value might indicate a new exposure for a visitor to an 
endemic region, IgG seropositivity in Singapore, where scrub typhus is endemic, likely reflects prior exposure to the organ-

\section{TABLE 1. Diagnostic Criteria for Hemophagocytic} Lymphohistiocytosis

The diagnosis of HLH may be established if either A or B is fulfilled.

A. A molecular diagnosis consistent with HLH is made (eg, mutations in PRF1, MUNC 13-4, STX11, SH2D1A)

\begin{tabular}{l}
\hline \multicolumn{1}{c}{ or } \\
\hline B. Diagnostic criteria for HLH are fulfilled ( $\geq 5$ of 8 must be present) \\
\hline 1. Fever, $\geq 38.5^{\circ} \mathrm{C}^{\mathrm{a}}$ \\
\hline 2. Splenomegaly \\
\hline 3. Cytopenias (affecting 2 lineages in peripheral blood) $)^{\mathrm{a}}$ \\
• Hemoglobin $<9 \mathrm{~g} / \mathrm{L}(<10 \mathrm{~g} / \mathrm{L}$ in infants $<4$ wk old) \\
• Platelets $<100 \times 10^{9} / \mathrm{L}$ \\
• Neutrophils $<1 \times 10^{9} / \mathrm{L}$ \\
\hline 4. Hypertriglyceridemia and/or hypofibrinogenemia ${ }^{a}$ \\
• Fasting triglycerides $\geq 3.0$ mmol/L \\
- Fibrinogen $\leq 1.5 \mathrm{~g} / \mathrm{L}$ \\
\hline 5. Hemophagocytosis in bone marrow or spleen or lymph nodes ${ }^{\mathrm{a}}$ \\
\hline 6. Low or absent natural killer cell activity \\
\hline 7. Ferritin $\geq 500 \mathrm{ng} / \mathrm{mL} \mathrm{L}^{\mathrm{a}}$ \\
\hline 8. Soluble $\mathrm{CD} 25$ (soluble interleukin 2 receptor), $\geq 2400 \mathrm{U} / \mathrm{mL}$ \\
\hline aPresent in patient. \\
NOTE: Abbreviation: HLH, hemophagocytic lymphohistiocytosis. \\
\hline
\end{tabular}

ism. Diagnosing an acute scrub typhus infection in a patient in an endemic region requires PCR testing. The skin biopsy results highlight the importance of understanding test characteristics. A skin biopsy specimen must be adequate in order to draw valid and accurate conclusions. In this case, the initial skin biopsy was superficial, and the specimen inadequate, but the test was not "negative." In the diagnostic skin biopsy, deeper tissue was sampled, and panniculitis (inflammation of subcutaneous fat), which arises in inflammatory, infectious, traumatic, enzymatic, and malignant conditions, was identified. An adequate biopsy specimen that contains subcutaneous fat is essential in making this diagnosis. ${ }^{2}$

This patient eventually manifested several elements of hemophagocytic lymphohistiocytosis (HLH), a syndrome of excessive inflammation and resultant organ injury relating to abnormal immune activation and excessive inflammation. $\mathrm{HLH}$ results from deficient down-regulation of activated macrophages and lymphocytes. ${ }^{3}$ It was initially described in pediatric patients but is now recognized in adults, and associated with mortality as high as $50 \% .^{3}$ A high ferritin level ( $>2000 \mathrm{ng} / \mathrm{mL}$ ) has $70 \%$ sensitivity and $68 \%$ specificity for pediatric HLH and should trigger consideration of HLH in any age group. ${ }^{4}$ The diagnostic criteria for HLH initially proposed in 2004 by the Histiocyte Society to identify patients for recruitment into a clinical trial included molecular testing consistent with HLH and/or 5 of 8 clinical, laboratory, or his-

TABLE 2. HScore for Diagnosing Hemophagocytic Lymphohistiocytosis (HLH)

\begin{tabular}{|c|c|}
\hline Parameter & No. of Points (Criteria for Scoring) \\
\hline \multirow[t]{2}{*}{ Known underlying immunosuppression (eg, HIV positive or receiving long-term immunosuppressive therapy) } & 0 (no) \\
\hline & 18 (yes) \\
\hline \multirow[t]{3}{*}{ Temperature $\left({ }^{\circ} \mathrm{C}\right)$} & $0(<38.4)$ \\
\hline & 33 (38.4-39.4) \\
\hline & $49(>39.4)$ \\
\hline Organomegaly & $0($ no) \\
\hline \multirow{3}{*}{$\begin{array}{l}\text { No. of cytopenias (Defined as hemoglobin level of } \leq 9.2 \mathrm{gm} / \mathrm{dl} \text { and/or leukocyte count of } \leq 5000 / \mathrm{mm}^{3} \text { and/or } \\
\text { a platelet count of } \leq 110,000 / \mathrm{mm}^{3} \text { ) }\end{array}$} & 0 (1 lineage) \\
\hline & 24 (2 lineages) \\
\hline & 34 (3 lineages) \\
\hline \multirow[t]{3}{*}{ Ferritin $(\mathrm{ng} / \mathrm{mL})$} & $0(<2,000)$ \\
\hline & $35(2000-6000)$ \\
\hline & $50(>6000)$ \\
\hline \multirow[t]{2}{*}{ Fibrinogen $(g / L)$} & $0(>2.5)$ \\
\hline & $30(\leq 2.5)$ \\
\hline \multirow[t]{2}{*}{ Serum glutamic oxaloacetic transaminase (IU/L) } & $0(<30)$ \\
\hline & $19(\geq 30)$ \\
\hline \multirow[t]{5}{*}{ Hemophagocytosis features on bone marrow aspirate } & 0 (no) \\
\hline & 35 (yes) \\
\hline & Total >169: strongly consider HLH \\
\hline & Sensitivity: $93 \%$ \\
\hline & Specificity 86\% \\
\hline
\end{tabular}


topathologic features (Table 1 ). ${ }^{5} \mathrm{HS}$ core is a more recent validated scoring system that predicts the probability of HLH (Table 2). A score above 169 signifies diagnostic sensitivity of $93 \%$ and specificity of $86 \%{ }^{6}$

The diagnosis of HLH warrants a search for its underlying cause. Common triggers are viral infections (eg, EBV), autoimmune diseases (eg, systemic lupus erythematosus), and hematologic malignancies. These triggers typically stimulate or suppress the immune system. Initial management involves treatment of the underlying trigger and, potentially, immunosuppression with high-dose corticosteroids or cytotoxic agents (eg, etoposide). Primary HLH is an inherited immunodeficiency, and treatment often culminates in stem cell transplantation. ${ }^{5}$

In this case, SPTCL triggered HLH. SPTCL is a rare non-Hodgkin lymphoma characterized by painless subcutaneous nodules or indurated plaques (panniculitis-like) on the trunk or extremities, constitutional symptoms, and, in some cases, HLH. ${ }^{7-10}$ SPTCL is diagnosed by deep skin biopsy, with immunohistochemistry showing CD8-positive pathologic $\mathrm{T}$ cells expressing cytotoxic proteins (eg, granzyme B)., ${ }^{9,11}$ SPTCL can either have an alpha/beta T-cell phenotype (SPTCL-AB) or gamma/delta T-cell phenotype (SPTCL-GD). Seventeen percent of patients with SPTCL-AB and $45 \%$ of patients with SPTCL-GD have HLH on diagnosis. Concomitant HLH is associated with decreased 5-year survival. ${ }^{12}$

This patient presented with fevers and was ultimately diagnosed with HLH secondary to SPLTCL. His case is a reminder that not all diseases in the tropics are tropical diseases. In the diagnosis of a febrile illness, a broad evaluative framework and rigorous test results evaluation are essential- no matter where a patient lives or visits.

\section{KEY TEACHING POINTS}

- A febrile illness acquired in the tropics is not always attributable to a tropical infection.

- To avoid diagnostic error, weigh positive or negative test results against disease features, patient epidemiology, and test characteristics.

- HLH is characterized by fevers, cytopenias, hepatosplenomegaly, hyperferritinemia, hypertriglyceridemia, and hypofibrinogenemia. In tissue specimens, hemophagocytosis may help differentiate HLH from competing conditions.

- After HLH is diagnosed, try to determine its underlying cause, which may be an infection, autoimmunity, or a malignancy (commonly, a lymphoma).

Disclosure: Nothing to report.

\section{References}

1. Centers for Disease Control and Prevention. Destinations [list]. http://wwwnc. cdc.gov/travel/destinations/list/. Accessed April 22, 2016.

2. Diaz Cascajo C, Borghi S, Weyers W. Panniculitis: definition of terms and diagnostic strategy. Am J Dermatopathol. 2000;22(6):530-549.

3. Ramos-Casals M, Brito-Zerón P, López-Guillermo A, Khamashta MA, Bosch X. Adult haemophagocytic syndrome. Lancet. 2014;383(9927):1503-1516.

4. Lehmberg K, McClain KL, Janka GE, Allen CE. Determination of an appropriate cut-off value for ferritin in the diagnosis of hemophagocytic lymphohistiocytosis. Pediatr Blood Cancer. 2014;61(11):2101-2103.

5. Henter JI, Horne A, Aricó M, et al. HLH-2004: diagnostic and therapeutic guidelines for hemophagocytic lymphohistiocytosis. Pediatr Blood Cancer. 2007;48(2):124-131.

6. Fardet L, Galicier L, Lambotte O, et al. Development and validation of the HScore, a score for the diagnosis of reactive hemophagocytic syndrome. Arthritis Rheumatol. 2014;66(9):2613-2620.

7. Aronson IK, Worobed CM. Cytophagic histiocytic panniculitis and hemophagocytic lymphohistiocytosis: an overview. Dermatol Ther. 2010;23(4):389-402.

8. Willemze R, Jansen PM, Cerroni L, et al; EORTC Cutaneous Lymphoma Group. Subcutaneous panniculitis-like T-cell lymphoma: definition, classification, and prognostic factors: an EORTC Cutaneous Lymphoma Group study of 83 cases. Blood. 2008;111(2):838-845.

9. Kumar S, Krenacs L, Medeiros J, et al. Subcutaneous panniculitic T-cell lymphoma is a tumor of cytotoxic T lymphocytes. Hum Pathol. 1998;29(4):397-403.

10. Salhany KE, Macon WR, Choi JK, et al. Subcutaneous panniculitis-like T-cell lymphoma: clinicopathologic, immunophenotypic, and genotypic analysis of alpha/beta and gamma/delta subtypes. Am J Surg Pathol. 1998;22(7):881-893.

11. Jaffe ES, Nicolae A, Pittaluga S. Peripheral T-cell and NK-cell lymphomas in the WHO classification: pearls and pitfalls. Mod Pathol. 2013;26(suppl 1):S71-S87.

12. Willemze R, Hodak E, Zinzani PL, Specht L, Ladetto M; ESMO Guidelines Working Group. Primary cutaneous lymphomas: ESMO clinical practice guidelines for diagnosis, treatment and follow-up. Ann Oncol. 2013;24(suppl 6): vi149-vi154. 